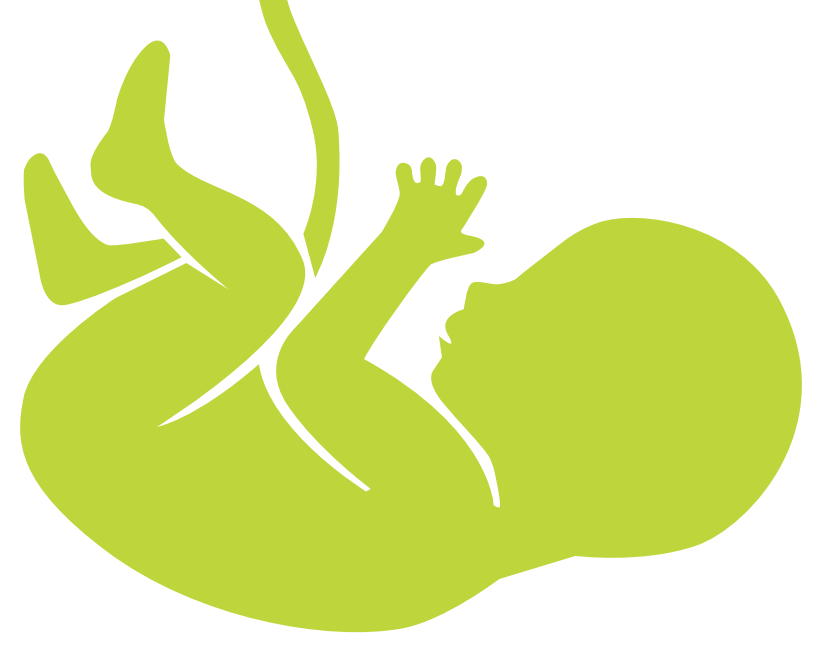

\title{
SISTEMA DE RECONHECIMENTO DE IMAGENS PARA AVALIAÇÃO DO MOVIMENTO TORACOABDOMINAL EM RECEM-NASCIDOS
}

IMAGE RECOGNITION SYSTEM FOR EVALUATING THORACOABDOMINAL MOVEMENTS IN NEWBORNS

\author{
Heitor Bernardino de Oliveira \\ Universidade Federal do Rio Grande do Norte - Campus Universitário Lagoa Nova CEP 59078-900 \\ Natal/RN - Brasil heitor@neuro.ufrn.br \\ Silvana Alves Pereira \\ Universidade Federal do Rio Grande do Norte - Campus FACISA - Rua Vila Trairi, S/N - Centro \\ CEP 59200-000 | Santa Cruz/RN - Brasil apsilvana@gmail.com \\ Bárbara Emmily Cavalcanti Vale \\ Universidade Federal do Rio Grande do Norte - Campus FACISA - Rua Vila Trairi, S/N - Centro \\ CEP 59200-000 | Santa Cruz/RN - Brasil babinha.a@hotmail.com \\ Danilo Alves Pinto Nagem \\ Universidade Federal do Rio Grande do Norte - Campus Universitário Lagoa Nova CEP 59078-900 \\ Natal/RN - Brasil danilo.nagem@gmail.com
}
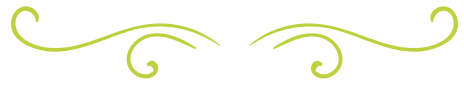

\section{RESUMO}

Este projeto tem sido desenvolvido em parceria com a Faculdade de Ciências da Saúde do Trairí (FACISA - UFRN) com o objetivo de otimizar a avaliação dos movimentos toracoabdominal em recém-nascidos, depois de receber manobra de fisioterapia respiratória. Em recém-nascidos, não existe nenhum método eficaz para avaliar a cinemática respiratória. A técnica tradicional para a medição do volume respiratório, chamado pletismografia, não é viável para avaliações clínicas de recém-nascidos. Alguns estudos propõem uma solução diferente para esse problema; usando imagens laterais do recém-nascido e processando essas imagens em software baseado em vetor, como CorelDraw ou AutoCAD, no qual o usuário tem que adicionar manualmente uma curva sobre a região toracoabdominal e delimitar as áreas desejadas. Em relação a esses programas, cada imagem é analisada individualmente, a área do foco é calculada e convertida para $\mathrm{cm}^{2}$, por aplicação de uma escala apropriada, a qual é derivada a partir de um valor de comprimento padrão na imagem. Após a análise de todas as imagens, o ponto máximo e mínimo do movimento respiratório é adquirido, proporcionando dados suficientes para avaliar a eficiência da manobra. No entanto, esse processo é lento e impreciso, porque depende da eficiência do usuário nos referidos softwares. Nesse contexto, este projeto foi desenvolvido, como um primeiro método funcional e automatizado para determinar o volume toracoabdominal a partir de imagens 2D de recém-nascidos. Desenvolvido usando caixas de ferramentas de processamento de imagem do MATLAB, o algoritmo é capaz de identificar os pontos de referência e de 
demarcar a curvatura do tórax automaticamente. Dessa forma, a aplicação foi capaz de processar e quantificar, sequencialmente, um conjunto maior de imagens, utilizando sempre o mesmo padrão, que determina as zonas mínima e máxima do tórax e abdômen durante todo o movimento respiratório de recém-nascidos, antes e após a aplicação das manobras de fisioterapia respiratória.

PALAVRAS-CHAVE: reconhecimento de imagem, cinemática respiratória, Mecânica respiratória. Fotogrametria. Recém-nascido

\section{ABSTRACT}

This project has been develop in partnership with Trairís Health Science Faculty (FACISA UFRN ) aiming to optimize the evaluation of thoracoabdominal movements in newborns, after receiving physiotherapeutic respiratory maneuver. However, in newborns, there is no effective method to assess respiratory kinematics. The traditional technique for respiratory volume measurement, called plethysmography, is not viable for clinical assessments in neonates. Some papers propose a different solution for this problem; by using lateral pictures of the newborn and processing those images in vector-based software, like CorelDraw or AutoCAD, where the user has to manually add a curve over the thoracoabdominal region to delimitate the wanted areas. On these softwares, each picture has to be individually analyzed, the focus area has to be calculated and converted to $\mathrm{cm}^{2}$, by applying an appropriated scale, which is derived from a standard length value in the image. After compiling all the images, the maximum and minimum point of the respiratory motion is acquired, providing enough data to evaluate the efficiency of the maneuver. Nevertheless, this process is slow and inaccurate, because it relies on user's proficiency on said softwares. In this context, this project was developed, as a first functional and automated method to determine the thoracoabdominal volume from 2D images of neonates. Developed using MATLAB's image processing toolboxes, the algorithm is capable of identifying the reference points and demarcating the thorax curvature automatically. This way, the application was able to process and quantify, sequentially, a larger set of images, always using the same standard, determining the maximum and minimum areas of the thorax and the abdomen throughout the entire respiratory motion of newborns, before and after applying the physiotherapeutic respiratory maneuver.

KEYWORDS: Image recognition, respiratory kinematics, Respiratory Mechanics. Photogrammetry. Infant newborn

\section{INTRODUÇÃO}

A cinemática do tórax e do abdômen durante o processo de respiração tem uma relação importante com a ventilação alveolar (BAVIS; MITCHELL, 2008). No entanto, avaliar a configuração toracoabdominal em recém-nascidos não é trivial. $\bigcirc$ método mais tradicional para a medição do volume respiratório é a pletismografia, que usa máquinas muito caras. Esse método consiste numa câmara fechada, na qual a pressão do sistema é medida e o volume do pulmão pode ser calculado usando a Lei de Boyle (BARRETO; CAVALAZZI, 2002).

Um método alternativo para avaliar esse processo é a fotogrametria, que é a ciência de fazer medições a partir de fotografias. Nesse caso, esse método é mais acessível para a avaliação clínica em comunidades de entrada baixas, e tem uma boa reprodutibilidade em neonatologia. A fotogrametria provou ser de grande valor para a aquisição de medições e informações numéricas usadas em aplicações clínicas em muitos campos da medicina (RICIERI, 2008).

O grupo de pesquisa da Faculdade de Ciências da Saúde do Trairí tem usado esse método para avaliar as melhorias da expansão torácica em recém-nascidos, depois de receber manobras respiratórias de fisioterapia. Para tanto, eles usaram imagens laterais do recém-nascido e processaram cada imagem individualmente no AutoCAD (PEREIRA et al., 2014), como pode ser observado na (Figura 1). Embora esse método seja eficaz, é demorado, pois cada imagem leva cerca 
de 25 minutos para processamento. Para ter certeza de obter o pico e a base do movimento respiratório, é necessário analisar ao menos sete imagens para cada bebê antes e sete após a manobra, o que resulta em quase 6 horas de trabalho para cada bebê (PEREIRA et al., 2014). O principal objetivo desse projeto é criar um algoritmo capaz de fazer esta tarefa automaticamente.

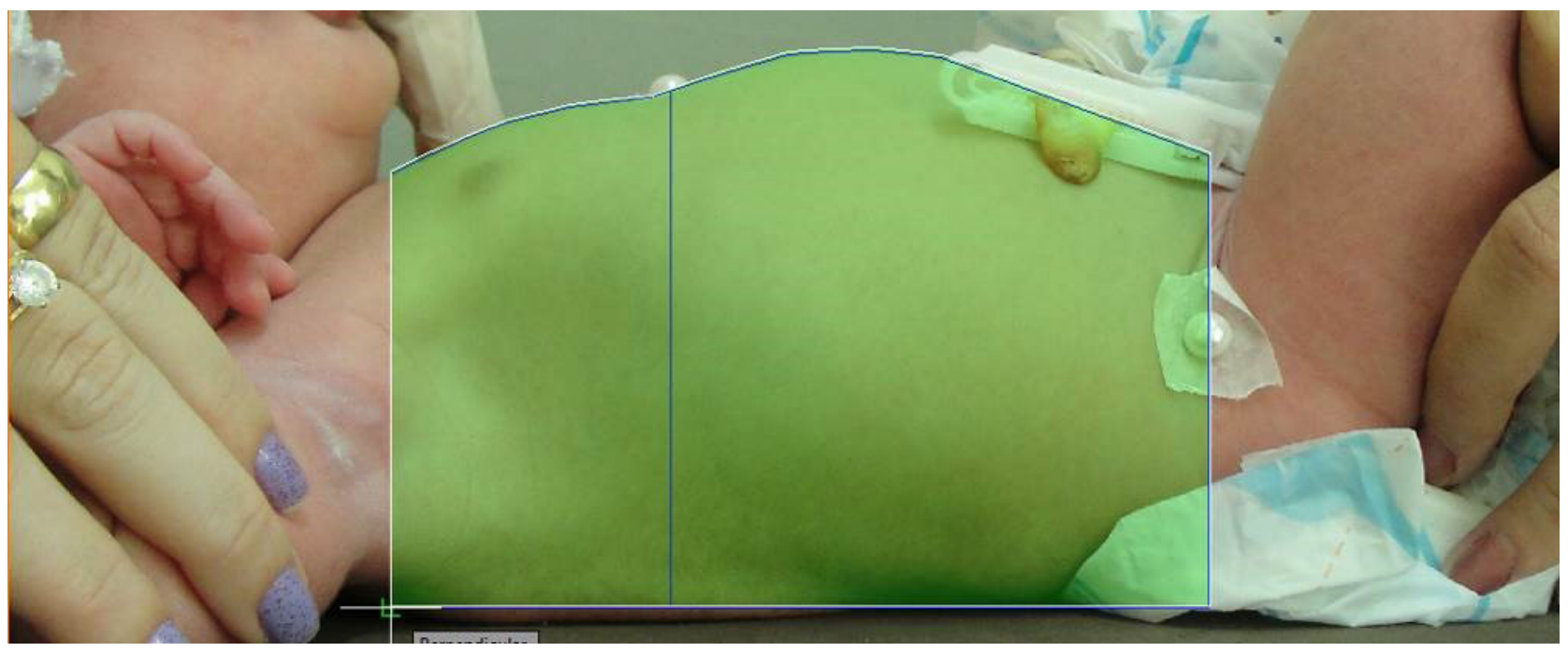

Figura 1 - Padronização para retirada da imagem pelo software AUTOCAD. Fonte: Autoria própria.

\section{METODOLOGIA}

Para garantir fotos que possam ser corretamente reconhecidas pelo software e cujos valores de medidas sejam coerentes para a comparação pré e pós-manobra, é preciso definir um padrão de procedimentos para a preparação do local e do bebê. Uma parte do procedimento que já era feita anteriormente e foi mantida é o posicionamento da câmera. Não é preciso definir um local com precisão para colocar a câmera, porém é importante que ela esteja no mesmo lugar para o mesmo bebê. A sequência do experimento é: posicionamento da câmera; fotos pré-manobra; manobras de fisioterapia; fotos pós-manobras. Durante as manobras de fisioterapia, a câmera é mantida no mesmo local das imagens pré-manobra, essa sequência, garante a comparação dos valores das áreas medidas. (Figura 2).

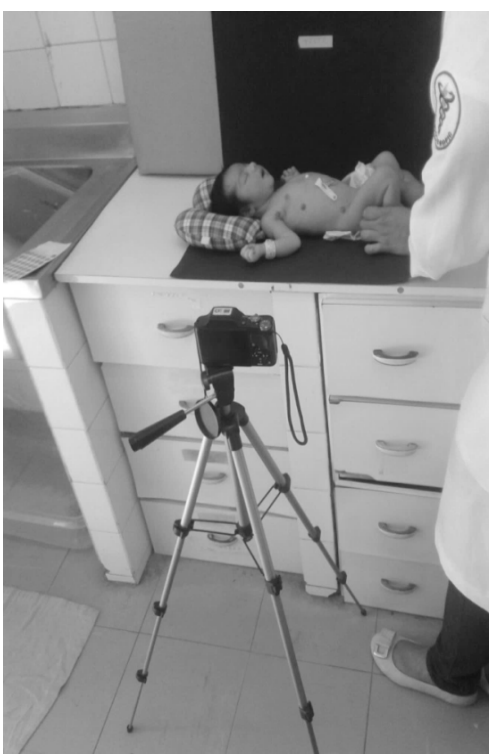

Figura 2 - Representação do cenário e posicionamento da câmera e do bebê durante a aplicação da manobra e extração das imagens, pré e pós manobra. Fonte: Autoria própria. 
$\mathrm{Na}$ análise do AUTOCAD, foram alocados dois marcadores em alto relevo na cor pérola, uma na posição das espinhas ilíacas ântero-superiores, para delimitar a região abdominal, e um segundo na posição do apêndice xifoide, para delimitar a região torácica. O contorno da parede superior do tórax era realizado manualmente pelo avaliador das imagens. Como pode ser visto na Figura 1.

$\mathrm{Na}$ análise pelo MATLAB foi acrescido um terceiro marcador, na cor vermelha, localizado no nível da incisura jugular do manúbrio esternal, projetado lateralmente. Esses três marcadores delimitam toda a área a ser estudada e excluem a necessidade de um contorno de área manual. A posição do bebê e marcadores são definidos, conforme RICIERI 2009 (FREITAS et al., 2007) como na (Figura 3).

A cor vermelha dos marcadores resulta um maior contraste entre marcador e pele do bebê; garantindo, assim, o reconhecimento da área a ser analisada. Outro problema de contraste encontrato foi entre a parede de fundo e a pele do bebê, que não poderia causar ambiguidades no reconhecimento da borda, gerando uma área diferente da real. Portanto, definiu-se a necessidade de utilizar um fundo preto. $\mathrm{Na}$ parte inferior da imagem são feitas duas marcas pretas, distantes 3 centímetros, utilizadas para converter as medidas de pixels para centímetros (Figura 3).

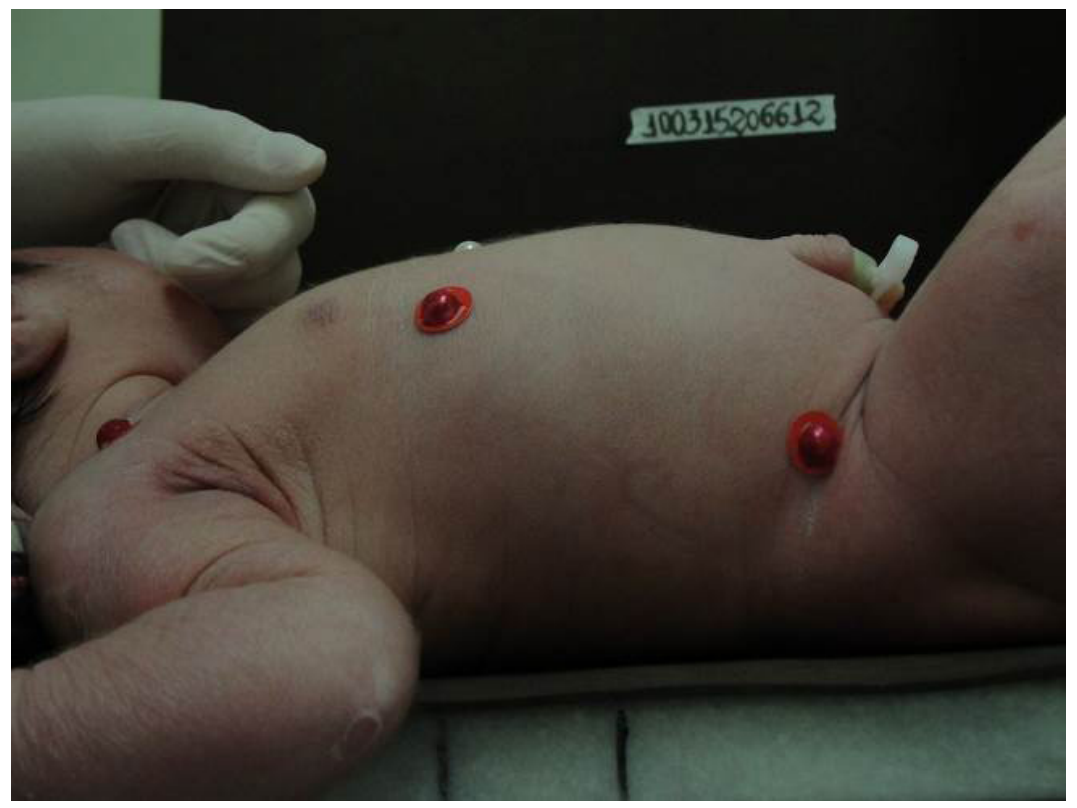

Figura 3 - Nova padronização para retirada da imagem pelo software MATLAB Fonte: Autoria própria.

\section{RESULTADOS}

O programa recebe como entrada um conjunto de fotos tiradas seguindo o padrão definido. Primeiramente, é feita a rotação da imagem, para garantir que as costas do bebê estejam na horizontal, dessa forma, facilita-se a delimitação das áreas analisadas e corrige-se algum possível erro no padrão de distância marcado. Essa rotação é feita com o usuário clicando dois pontos entre as costas do bebê e a mesa, como a câmera não se mexe, esse processo só é feito uma vez para cada conjunto de fotos do mesmo bebê. Após a rotação, o usuário seleciona dois pontos no centro das retas pretas, definindo o valor da escala que converte de pixel para centímetro.

Em segundo lugar, é feito o reconhecimento dos pontos vermelhos, que é feito por meio da subtração entre duas imagens, a primeira é somente a componente vermeIha da foto original, e a segunda é a imagem original em escala de cinza. Na sequência, 
aplica-se um filtro de média para reduzir o ruído e transformar a figura em imagem binária usando um threshold adequado, obtendo apenas uma imagem toda preta, com manchas brancas no local onde estavam as referências. Esse processo pode ser visto a seguir (Figura 4).
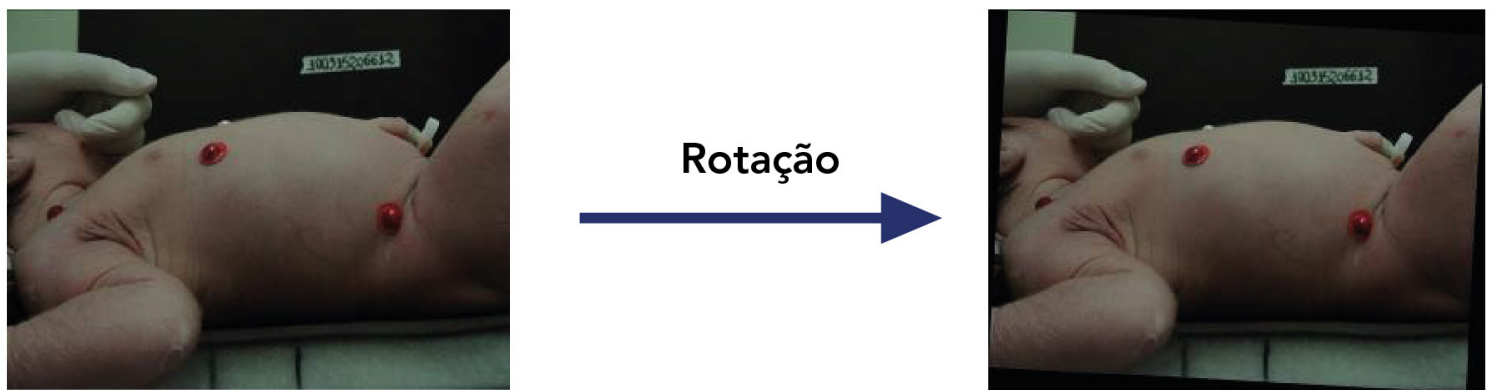

Subtração
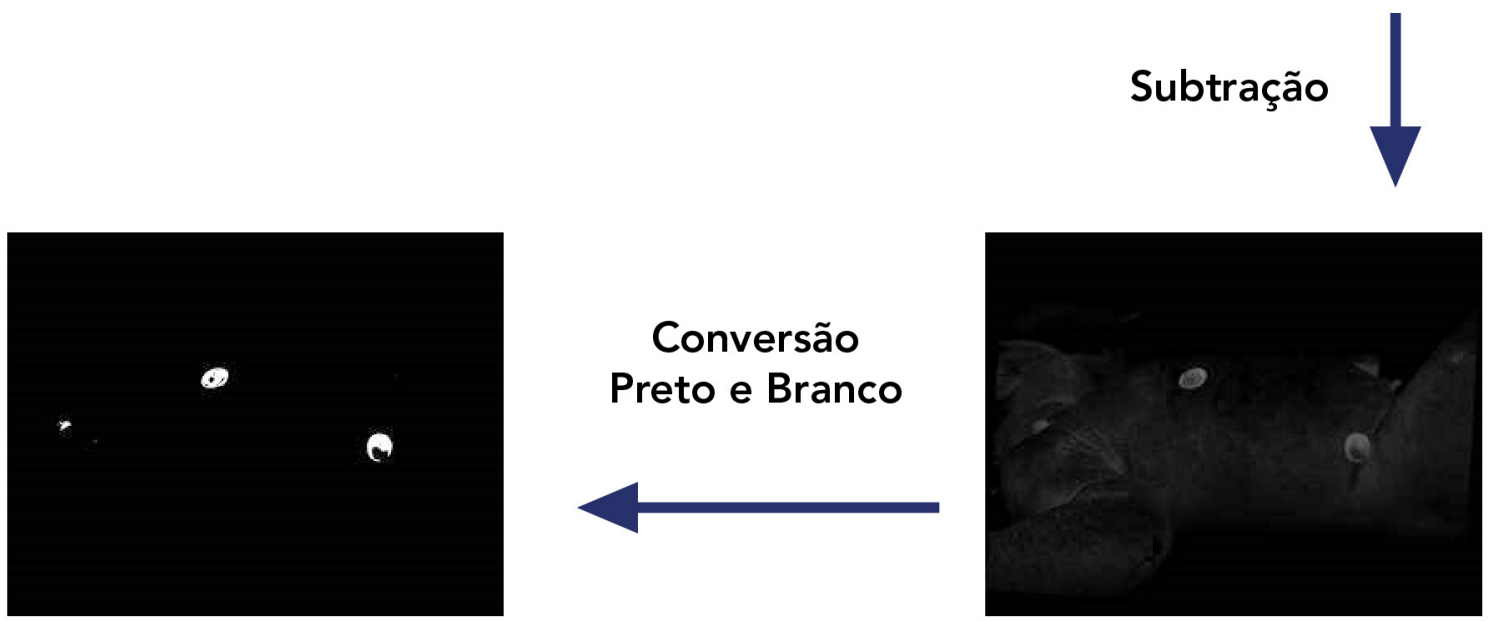

Figura 4 - Primeira Sequência do Processamento da Imagem.

Fonte: Autoria própria.

Utilizando a função Blob Analysis do pacote vision do MATLAB, estipulam-se os parâmetros para reconhecimento de regiões binárias conectadas. Definindo um tamanho mínimo para as manchas brancas encontradas, eliminam-se pequenas regiões vermelhas que podem surgir, devido ao tom da pele do bebê, achando o centro apenas das maiores áreas brancas, que representam as marcas vermelhas (FREITAS et al., 2007).
Agora, o programa encontra a borda entre a barriga do bebê e a parede. Voltando a imagem original rotacionada, aplica-se um filtro para aumentar o constrate da imagem e, em seguida, aplica-se um filtro de média para suavizar a transição entre o fundo preto e o corpo do bebê. Essa nova imagem é convertida em imagem binária, obtendo uma imagem com a borda entre o corpo e o fundo bem definida. 

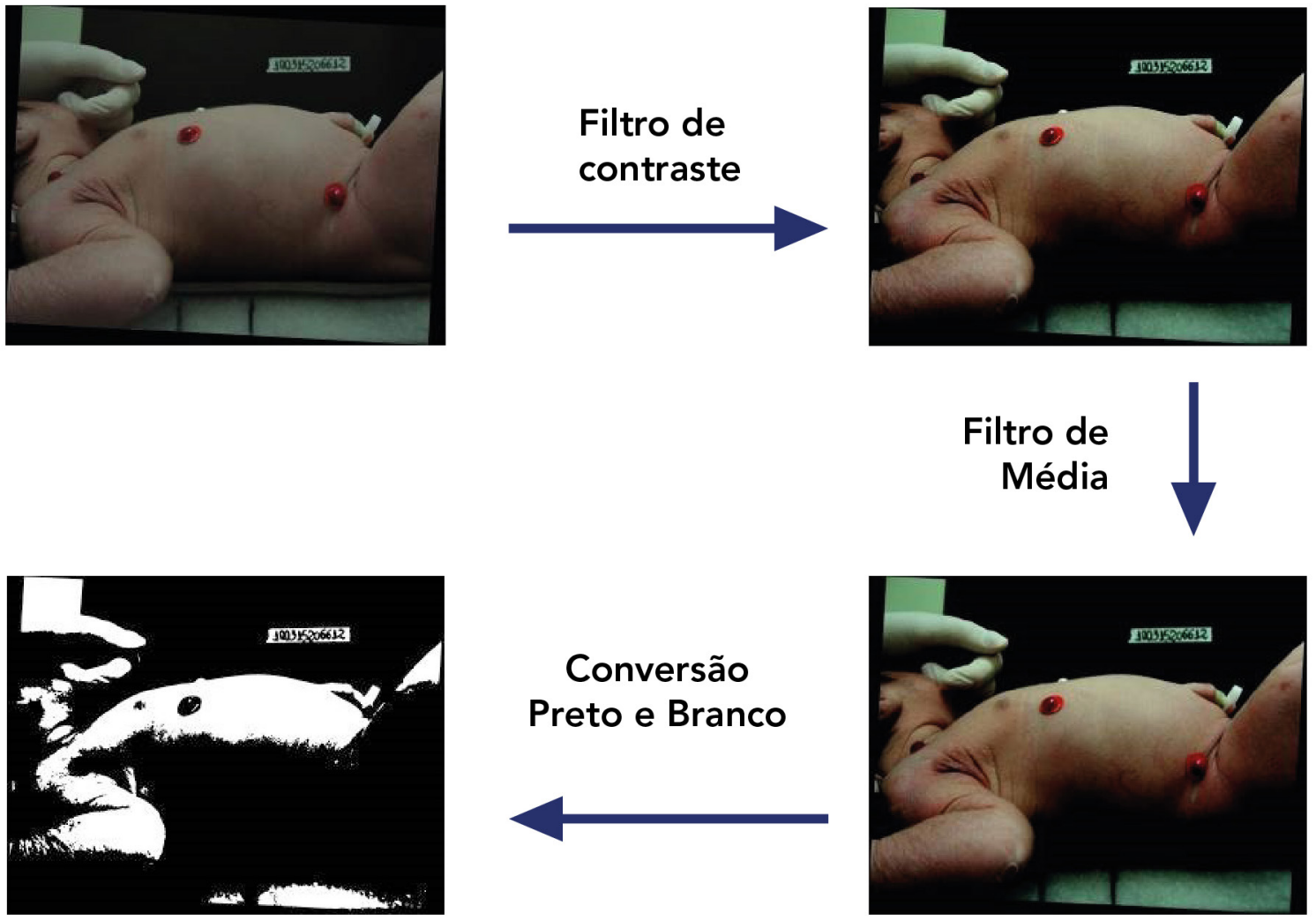

Figura 5 - Segunda Sequência do Processamento da Imagem.

Fonte: Autoria própria.

Com a imagem em preto e branco, faz-se uma varredura na coluna do centro do marcador localizado no apêndice xifoide, até encontrar o corpo do bebê e, na sequência, segue-se fazendo o mesmo para o lado direito até o limite dado pelo centro do marcador da direita, adquirindo a região do abdomen do bebê. Fazendo a varredura para o lado esquerdo, obtém-se a região torácica (Figura 6 e 7).

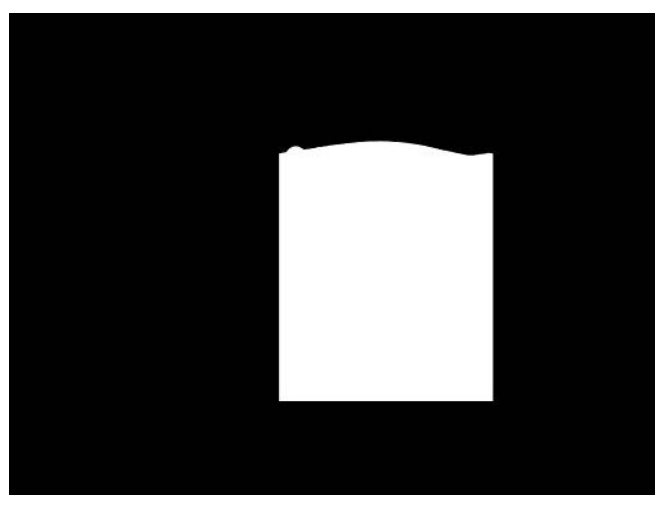

Figura 6 - Área do abdômen.

Fonte: Autoria própria.

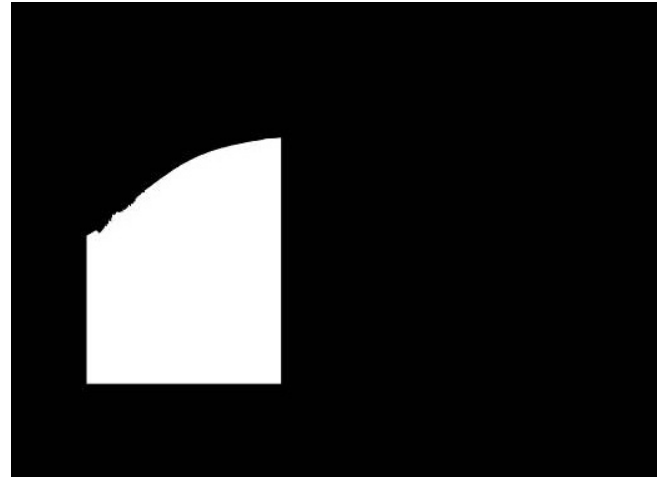

Figura 7 - Área do tórax.

Fonte: Autoria própria.

Nessas imagens, calculam-se as duas áreas (em pixel quadrados) que são utilizadas na avaliação da cinemática respiratória. Utilizando essas imagens como máscara, obtém-se uma imagem semelhante à construída no AutoCAD (Figura 8). 


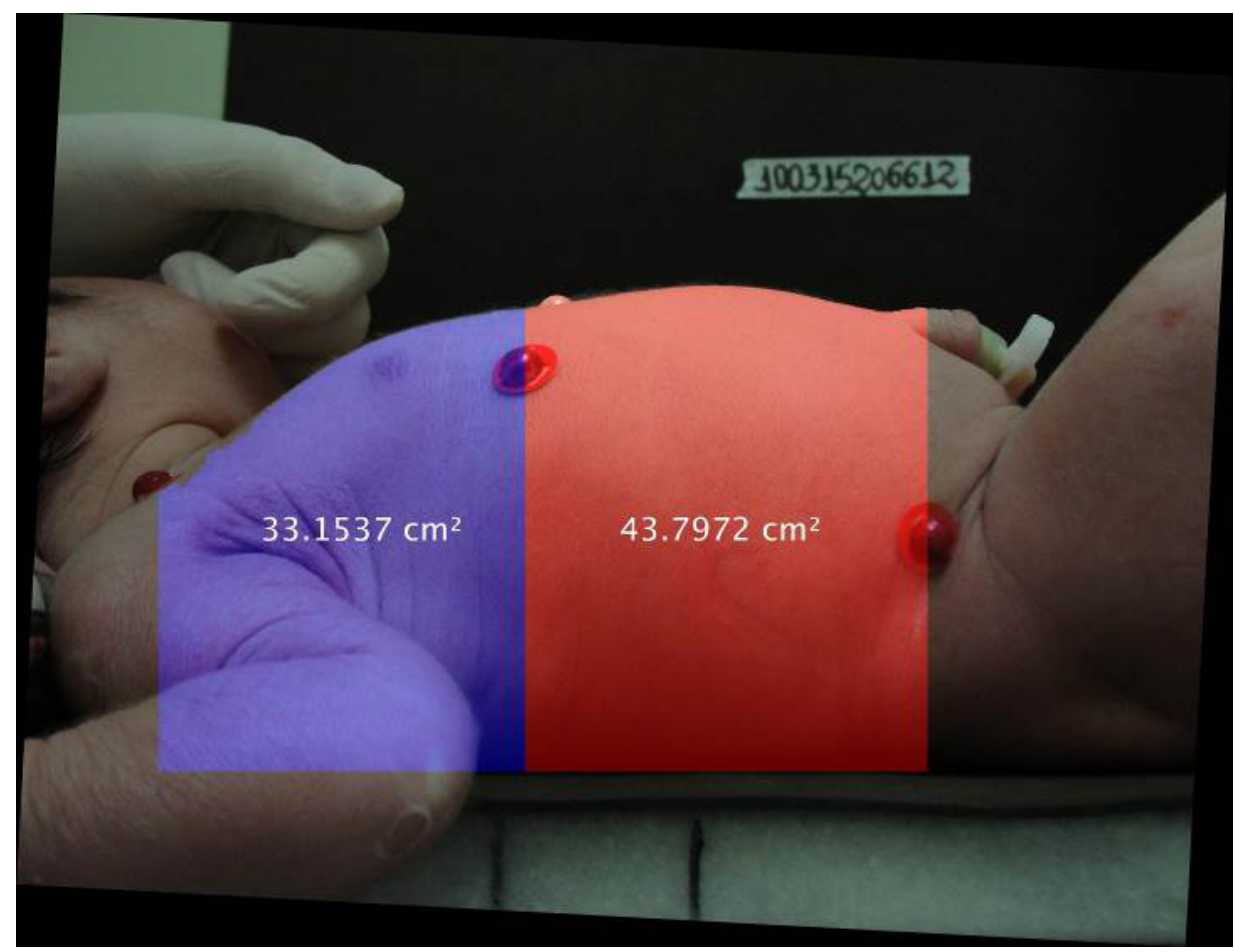

Figura 8 - Imagem de Saída do Programa.

Fonte: Autoria própria.

\section{DISCUSSÃO}

Quando comparado com os valores obtidos no processo anterior, o programa proposto mostrou-se consistente no cálculo da área, com valores mais precisos e menos sujeitos a variações inerentes do usuário. Para cada set de imagens, que corresponde a 14 fotos do mesmo bebê, sete antes e sete depois da manobra, o programa demora em torno de 20 segundos para marcação dos pontos, para rotação da imagem e dos pontos e para definição da escala. Após isso, o programa demora em torno de 0.9 segundos para processar cada imagem e cerca de 0.4 segundos para salvá-la, resultando em $38.2 \mathrm{~s}$ para processar todas as imagens, processo que demorava, como citado anteriormente, 25 minutos por foto.

No período neonatal, a musculatura respiratória é enfraquecida, assim como os demais músculos esqueléticos (RICIERI; ROSÁRIO FILHO, 2009; BAVIS et al., 2007; MITCHELL; JOHNSON, 2003) e, dessa forma, manobras que comprimem a caixa torácica podem levar ao decréscimo das pressões inspiratórias e expiratórias (CARROLL, 2003). O conhecimento dessas importantes características torna o controle da ventilação, durante um programa de fisioterapia respiratória, um desafio. E o novo método proposto mostrou-se capaz de analisar, automaticamente, esse movimento da área toracoabdominal, o que possibilita um rol de marcações dentro da prática clínica neonatal.

Embora já exista todo esse conhecimento científico acerca da fisiologia neonatal e aplicabilidade das manobras de fisioterapia, eram necessários métodos onerosos para avaliação e verificação desses achados no sistema respiratório de recém-nascidos. ${ }^{4}$ Desse modo, a monitorização não invasiva, com ênfase na avaliação da parede torácica, proposta neste estudo, tem adquirido relevância nas mais diversas faixas etárias (RICIERI, 2008; PEREIRA et al., 2014; DINIZ et al., 2014; BARAÚNA et al, 2006).

Vários métodos de superfície, regidos por princípios físicos diferentes, eram utilizados na avaliação do contorno e movimento toracoabdominal durante a respiração, alguns desses não aplicados a neonatologia como a cirtometria (RICIERI; ROSÁRIO FILHO, 2008), por exemplo, outros são onerosos e muitas vezes direcionados a laboratórios de pesquisa como a pletismografia por indutância (DELLACA et al., 2010). Entretanto, o método proposto, este artigo 
articula um modelo, 2D, que se destaca como método avaliativo do compartimento toracoabdominal em situações diversas na neonatologia. Modelos 2Ds já foram propostos por outros experimentos em situações diversas, (RICIERI, 2008; PEREIRA et al., 2014; FREITAS et al., 2007; DINIZ et al., 2014; BARAÚNA et al, 2006), todavia, esse foi o primeiro modelo criado para avaliação automática da área toracoabdominal em recém-nascidos.

A avaliação do compartimento toracoabdominal proposto neste estudo forneceu medidas quantitativas da área pulmonar, pelos movimentos respiratórios detectados por fotografias, permitindo a avaliação do movimento e expansibilidade toracoabdominal durante a respiração espontânea e após a realização de manobras de fisioterapia respiratória em menos de 40 segundos.

Outro fator importante é que, anteriormente, as imagens eram feitas utilizando a metodologia do teste cego, ou seja, o avaliador que fazia o processamento das imagens não sabia se o bebê tinha sido submetido ou não às manobras, a fim de evitar possíveis manipulações dos dados. No novo modelo automatizado, elimina-se a necessidade de tal procedimento, uma vez que o usuário não interfere no resultado medido. 


\section{REFERÊNCIAS}

BARAÚNA, M. A. et al. Avaliação do equilíbrio estático em indivíduos amputados de membros inferiores através da Biofotogrametria Computadorizada. Revista Brasileira de Fisioterapia, v. 10, n. 1, p. 83-90, 2006.

BARRETO, S. S. M.; CAVALAZZI, A. C. Determinações dos volumes pulmonares. Métodos de mensuração dos volumes pulmonares. J Pneumol., v. 28, supl. 3, out. 2002.

BAVIS, R. W.; MITCHELL, G. S. Long-term Effects of the Perinatal Environment on Respiratory Control. Journal of Applied

Physiology, v. 104, n. 4, p. 1220-1229, apr. 2008.

BAVIS, R. W. et al. Respiratory plasticity in response to changes in oxygen supply and demand. Integrative and Comparative Biology, v. 47, n. 4, p. 532-551, 2007.

CALDEIRA, V. S. et al. Precisão e acurácia da cirtometria em adultos saudáveis. Jornal Brasileiro de Pneumologia, v. 33, n. 5, p. 519-526, 2007.

CARROLL, J. L. Plasticity in Respiratory Motor Control Invited Review: Developmental plasticity in respiratory control. Journal of Applied Physiology, v. 94, p. 375-389, 2003.

DELLACA, R. L. et al. Measurement of total and compartmental lung volume changes in newborns by optoelectronic plethysmography. Pediatric Research, v. 67, n. 1, p. 11-16, 2010.

DINIZ, N. F. et al. Assessment of the Effects of Manual Chest Compression Technique on Atelectasis in Infants: A Randomized Clinical Trial. International Journal of Clinical Medicine, v. 5, p. 507-513, 2014.

FREITAS, R. F. et al. Algoritmos para segmentação da pele utilizando modelos de cores RGB em ambiente MATLAB/ SIMULINK. Conexões - Ciência e Tecnologia, v. 1, p. 65-71, 2007.

MITCHELL, G. S.; JOHNSON, S. M. Plasticity in Respiratory Motor Control Invited Review: Neuroplasticity in respiratory motor control. Journal of Applied Physiology, v. 94, p. 358-374, 2003.

PEREIRA, Silvana Alves et al. Avaliação não invasiva da mecânica respiratória toracoabdominal em recémnascidos: um modelo fotogramétrico. In: SIMPÓSIO INTERNACIONAL DE FISIOTERAPIA CARDIORRESPIRATÓRIA E FISIOTERAPIA EM TERAPIA INTENSIVA. 18., 2014, Salvador. Anais... São Paulo: Assobrafir, 2014. v. 5. p. 92-92. 
RICIERI, D. V. Princípios processuais da biofotogrametria e sua adaptação para medidas em estudos sobre movimentos respiratórios toracoabdominais. 2008. 188 f. Tese (Doutorado) Universidade Federal do Paraná, Curitiba, 2008.

RICIERI, D. V.; ROSÁRIO FILHO, N. A. Efetividade de um modelo fotogramétrico para a análise da mecânica respiratória toracoabdominal na avaliação de manobras de isovolume em crianças. Jornal Brasileiro de Pneumologia, São Paulo, v. 35, n. 2, feb. 2009.

RICIERI, D. V.; ROSÁRIO FILHO, N. A. Impacto de fatores externos sobra a mecânica respiratória avaliada por um modelo fotogramétrico específico: biofotogrametria. Jornal Brasileiro de Pneumologia, v. 34, n. 9, p. 702-706, 2008. 